\title{
Malignancies in Autoimmune Rheumatic Diseases - A Mini-Review
}

\author{
Zoltán Szekanecz $^{\mathrm{a}}$ Éva Szekanecz $^{\mathrm{b}}$ Gyula Bakóc $^{c}$ Yehuda Shoenfeld $^{\mathrm{d}}$ \\ ${ }^{a}$ Department of Rheumatology, Institute of Medicine, ${ }^{b}$ Department of Oncology and ${ }^{c}$ Division of Geriatry, \\ Third Department of Medicine, Medical and Health Science Center, University of Debrecen, Debrecen, Hungary; \\ ${ }^{d}$ Department of Medicine B and Center for Autoimmune Diseases, Sheba Medical Center, Tel-Hashomer, Israel
}

\section{Key Words \\ Malignancies • Paraneoplasia • Biological therapy • \\ Secondary tumors $\cdot$ Tumor-associated antigens}

\begin{abstract}
Paraneoplastic symptoms, caused by a malignancy, but not directly related to invasion by the tumor or its metastases, are the result of a wide variety of tumor-derived biologic mediators like hormones, peptides, antibodies, cytotoxic lymphocytes, autocrine and paracrine mediators. Systemic inflammatory rheumatic diseases, such as rheumatoid arthritis, systemic lupus erythematosus, scleroderma or dermatomyositis, may increase the risk for the development of malignancies, predominantly lymphoproliferative disorders. Immunosuppressive drugs and biological agents may also be carcinogenic. However, sustained inflammatory activity seems to be the primary risk factor for malignancies in autoimmune diseases. Tumor-associated antigens may be produced by inflammatory cells and their production may be increased in rheumatoid arthritis and other autoimmune diseases.

Copyright $\odot 2010$ S. Karger AG, Basel
\end{abstract}

\section{Introduction}

Malignancies have been associated with autoimmune rheumatic diseases. There are various aspects of the association of rheumatic diseases with cancer: (1) musculoskeletal symptoms and syndromes may develop as paraneoplastic diseases during the presence of malignancies; (2) there may be an increased incidence of tumors in certain systemic inflammatory and autoimmune diseases; (3) someimmunosuppressive agents, including DMARDs, may increase the risk for cancer; and (4) circulating tumor markers may be elevated in the sera of patients with arthritis. Primary or metastatic tumors developing within the musculoskeletal system will not be discussed in this paper.

Although autoimmune disease usually develops in younger patients, malignancies usually occur in elderly patients with long disease duration. Furthermore, as discussed later, older age may be associated with higher risk for cancer development. Therefore, this issue may have significant relevance for the elderly.

\section{Musculoskeletal Paraneoplasias}

\section{Paraneoplastic Rheumatic Syndromes}

Paraneoplastic rheumatic syndromes include syndromes resembling connective tissue diseases, vasculitis, arthropathies, bone disorders, diseases of the skin and

\section{KARGER}

Fax +41613061234

E-Mail karger@karger.ch

www.karger.com (c) 2010 S. Karger AG, Basel

0304-324X/11/0571-0003\$38.00/0

Accessible online at:

www.karger.com/ger
Zoltán Szekanecz, MD, PhD

Department of Rheumatology, Institute of Medicine

Medical and Health Science Center, University of Debrecen

Nagyerdei str. 98, HU-4032 Debrecen (Hungary)

Tel. +36 52255 091, Fax +36 52414 489, E-Mail szekanecz.zoltan@med.unideb.hu 
muscles, etc., which will be discussed briefly (reviewed in [1-5]) (table 1). Most of the reports are case studies and there is an immense need for larger international cohorts or registries. Moreover, if an autoimmune disease and malignancy develops within a short time, it may be difficult to distinguish an autoimmune paraneoplasia developing in a cancer patient from a secondary tumor occurring in an autoimmune disease.

\section{Connective Tissue Diseases}

Polymyositis and dermatomyositis (DM; including juvenile DM), amyopathic DM and inclusion body myositis may be associated with various types of cancer $[2,5,6]$. In a recent Hungarian cohort, nobody out of $154 \mathrm{PM}$ patients, but 12 of $63 \mathrm{DM}$ patients (19\%), had associated malignancy during follow-up [6]. Among cancer types, lung, breast, ovarian and nasopharyngeal cancer, as well as lymphoma have been associated with paraneoplastic myositis $[5,6]$. Paraneoplastic DM cases may exert more severe skin lesions and diaphragmatic involvement. In addition, DM do not respond to conventional immunosuppressive therapy (reviewed in [2, 4-6]).

Lupus-like syndrome in cancer patients includes polyserositis, Raynaud's phenomenon and a positive antinuclear antibody test. Ovarian cancer, breast cancer, mesothelioma and hairy cell leukemia have been associated with lupus-like disease $[1,2,4]$.

Scleroderma-like disease has been associated with lung, skin, breast and ovarian cancer. Anti-topoisomerase type 1 antibody was detected in numerous paraneoplastic cases $[5,7]$.

Late onset Raynaud's syndrome may be associated with gastrointestinal tumors, lung, ovarian or renal carcinoma, as well as lymphoproliferative diseases. The symptoms develop in elderly patients and feature asymmetric involvement with digital necrosis. Triggering factors include cryoglobulinemia, immune complex-induced vasospasm, hypercoagulability and vasculitis $[4,5$, 8].

\section{Vasculitis Syndromes}

Paraneoplastic polymyalgia rheumatica exerts atypical features, such as unilateral symptoms, unusually low $(<50 \mathrm{~mm} / \mathrm{h})$ or high $(>100 \mathrm{~mm} / \mathrm{h})$ erythrocyte sedimentation rate, and no response to corticosteroids. Polymyalgia rheumatica may be associated with colon, kidney, or lung cancer, or multiple myeloma $[2,4,9]$.

Paraneoplastic erythema nodosum persists for more than 6 weeks. These cases of erythema nodosum do not respond to corticosteroid or NSAID treatment. Only the
Table 1. Paraneoplastic syndromes

Connective tissue diseases

Polymyositis and dermatomyositis

Lupus-like syndrome

Scleroderma-like syndrome

Late-onset Raynaud's syndrome

Arthritides

Hypertrophic osteoarthropathy (secondary)

Carcinomatous polyarthritis (RA-like)

Relapsing polychondritis

RS3PE syndrome

Palmar fasciitis and polyarthritis

Vasculitis syndromes

Atypical polymyalgia rheumatica

Erythema nodosum

Cryoglobulinemic vasculitis

Skin and muscle diseases

Dermatomyositis

Lambert-Eaton syndrome

Palmar fasciitis

Panniculitis

Eosinophilic fasciitis

Metabolic diseases

Gout

Reflex sympathetic dystrophy

removal of the underlying tumor leads to full remission $[1,4,10]$.

Other types of vasculitis including polyarteritis nodosa, Wegener's granulomatosis, temporal arteritis or Churg-Strauss syndrome may develop in myelodysplasias, hairy cell leukemia and lymphoproliferative disorders. Paraneoplastic vasculitis may be a consequence of impaired reticuloendothelial function with reduced clearance of circulating antigens and immune complexes. The determination of ANCA does not help to identify paraneoplasia $[1,2,4]$.

\section{Arthropathies}

Carcinomatous polyarthritis is an atypical presentation of rheumatoid arthritis (RA). It generally develops in elderly patients, has an explosive onset and is often a seronegative asymmetric disease. The lower extremities are more often involved. Pathogenic factors include hormones, cytokines, peptides, antibodies and cytotoxic lymphocytes underlying synovitis. There is no evidence of tumor infiltration of the involved joints. Furthermore, joint radiographs tend to be normal. RA-like paraneoplasias have been associated with breast, colon, lung, ovari- 
an, gastric and esophageal cancer, as well as with lymphoproliferative diseases $[4,11]$.

Hyperthrophic osteoartropathy (HOA) is a prototype of tumor-induced paraneoplasia. The syndrome is the combination of clubbing of the fingers, pain, swelling and stiffness of peripheral joints. HOA is most often associated with lung cancer, lung metastases or pleural mesothelioma. Growth factors, including vascular endothelial and platelet-derived growth factor, have been implicated in the pathogenesis of tumor-associated HOA. The removal of the tumor results in the resolution of HOA symptoms in association with the reduction of circulating vascular endothelial growth factor levels [2, $4,12]$.

Relapsing polychondritis is a chronic inflammatory disease, with episodes of inflammation of cartilaginous tissues including the nose, ears, tracheobronchial tree and joints. It may be associated with myelodysplastic syndromes. Pathogenic factors include autoantibodies to type II collagen $[4,13]$.

Relapsing seronegative symmetric synovitis with pitting edema (RS3PE syndrome) may also occur in association with malignant diseases. The patients are elderly and generally males. The associated malignancy may be $\mathrm{T}$ cell lymphoma or myelodisplasia, or colon, lung, gastric, prostate or endometrial cancer [14].

Palmar fasciitis and polyarthritis present as palmar fasciitis ranging from diffuse globular swelling with warmth and erythema to typical Dupuytren's contractures, as well as atypical polyarthritis. Rheumatoid factor and antinuclear antibodies are negative. This syndrome may be associated with ovarian, endometrial, gastric, breast or prostate cancer, as well as chronic lymphocytic leukemia and Hodgkin's disease [2, 4].

\section{Metabolic Syndromes}

Hyperuricemia and gout have been described in association with disseminated carcinoma or lymphoproliferative disorders. Hyperuricemia may also present during cytotoxic treatment of the tumor. The severity of gout correlates with the extent of malignant disease, involvement of the liver, and the presence of hypercalcemia $[2,4]$.

Reflex sympathetic dystrophy (algoneurodystrophy) is most often associated with lung tumors; however, it may also develop in bowel, ovarian, pancreatic cancer and chronic myeloid leukemia. If no underlying trauma, stroke or myocardial infarction can be detected, screening for malignancy is necessary [4].

Malignancies and Autoimmunity
Table 2. Rheumatic diseases with increased risk for malignancies
Sjögren's syndrome

Rheumatoid arthritis

Systemic lupus

erythematosus

Systemic sclerosis

Dermatomyositis
Lymphoproliferative diseases

Lymphoproliferative diseases (cancer?)

Lymphoproliferative diseases

Lung, skin and esophageal cancer

Ovarian, lung and gastric cancer

\section{Skin and Muscle Diseases}

Polymyositis and DM are discussed above.

Eosinophil fasciitis is a scleroderma-like disorder associated with peripheral eosinophilia, presenting with sudden onset of unexplained hand pain, inflammatory fasciitis or palmar fibromatosis with or without digital contractures. The association between cancer and eosinophil fasciitis has been described predominantly in patients with lymphoproliferative diseases [2-4]. Palmar fasciitis associated with polyarthritis is discussed above.

Panniculitis, the inflammation of the subcutaneous adipose tissue, was observed in connection with pancreatic adenocarcinoma. As arthritis may also develop, some authors use the term 'pancreatic arthritis syndrome'. Pathogenic factors include fibrogenic growth factors and cytokines. Arthropathy is usually secondary to periarticular fat necrosis. Pathogenic factors may include the local action of trypsin and lipase, as well as the action of free fatty acids. Thus, the possibility of underlying pancreatic cancer should be considered in elderly patients with subcutaneous nodules in association with arthritis $[3,15]$.

Erythromelalgia is a syndrome of erythema, burning pain and warmth of the extremities. Association with myeloproliferative disorders has been described [2]. Pathogenesis includes arteriovenous shunting. In case of erythromelalgia, attention must be paid to any abnormalities in the blood count, such as leukocytosis, thrombocytosis and/or increased hemoglobin. Aspirin is highly effective $[4,16]$.

\section{Secondary Malignancies in Rheumatic Diseases}

\section{General Considerations}

There have been numerous reports of an association between rheumatic diseases and the development of tumors (table 2). Several factors including the autoimmune disease itself, common etiology between the rheumatic disease and malignancy, including genetic factors, virus- 
es (e.g. Epstein-Barr virus, retroviruses), smoking causing tissue necrosis, etc., have been implicated in the pathogenesis of tumor development. However, it is difficult to separate disease-related mechanisms from the potential oncogenic properties of immunosuppressive drugs used in these autoimmune-inflammatory diseases (reviewed in $[1,5,17,18])$. As discussed above, it may be difficult to differentiate secondary malignancies developing in an autoimmune patient and musculoskeletal paraneoplasias.

\section{Malignancies Associated with Connective Tissue Diseases}

\section{Sjögren's Syndrome}

The development of lymphoproliferative diseases in Sjögren's syndrome (SS) is the prototypic example of the increased risk of malignancy in a chronic autoimmune disease $[5,17]$. In recent large cohorts, the estimated odds ratio of lymphoma in SS was between 2.0 and 18.8. This risk, possibly due to better treatment and closer followup, has decreased with time, as in the late 1970s SS patients had an approximately 44 -fold risk of developing lymphoma. This is not a paraneoplastic syndrome, but rather chronic excessive salivary and lacrimal gland $\mathrm{B}$ cell stimulation and impaired B cell apoptosis that leads to tumorigenesis and clonal expansion of $\mathrm{B}$ cells.

Epstein-Barr virus has been implicated in chronic B cell proliferation and the development of lymphoma. In addition, chromosomal translocations may be present in SS patients with increased risk for lymphoma. Lymphomas including non-Hodgkin's lymphoma and specifically low-grade B cell lymphoma, as well as MALT lymphoma, Waldenström's macroglobulinemia, chronic lymphocytic leukemia and multiple myeloma develop in $4-10 \%$ of primary SS cases $[1,17,18]$. The B cell inhibitor anti-CD20 antibody, rituximab, was able to induce remission in 3 of 7 patients with MALT lymphoma associated with primary SS [19].

\section{Rheumatoid Arthritis}

Several investigators found an increased risk for the development of lymphoproliferative disorders and lung cancer, but a decreased risk for the development of colorectal malignancies in RA (reviewed in $[17,20]$ ). High long-term inflammatory activity in RA seems to have been associated with increased tumor risk with an odds ratio of 25.8 compared to low disease activity. In addition, cancer risk in RA may also be related to age and long- lasting disease. In large recent cohorts, the risk of lymphoma in RA patients was 1.4- to 3.9-times higher than in the general population. Patients with Felty's syndrome were found to have a 12 -fold increase in risk of lymphoma $[17,20]$. Also, B cell stimulation caused by chronic Epstein-Barr virus infection may be a pathogenic factor in RA-, as well as SS-associated lymphoma $[17,18]$. Smoking may be an important pathogenic factor in RA-associated lung cancer, as well as in RA itself [21]. Therefore, the pathogenic effects of smoking and RA in the development of lung cancer cannot be fully distinguished.

In a recent study, we assessed 516 RA patients undergoing follow-ups in our department for secondary malignancies. Although the number of RA patients with tumor is relatively small, we compared our cohort to the Health for All (HFA) database and calculated standard incidence ratios (SIR). We identified 13 cases of malignancy in the $516 \mathrm{RA}$ patients (2.5\%). The mean age of these patients at the time of the diagnosis of cancer was 61.8 years. Five patients died, four of them due to the underlying malignancy. The overall survival was 4.7 years. Six patients had lung cancer (SIR 10.9), two had follicular thyroid cancer (SIR 70.7), while the others had cutaneous B cell lymphoma (SIR 6.1), breast cancer (SIR 2.5), gall bladder cancer (SIR 18.5), colorectal cancer (SIR 2.2) and ovarian cancer (SIR 15.6). Thus, the overall SIR of all malignancies in RA was 1.12 (CI: $0.91-1.33$ ), varying between 2.2 and 70.7 among different tumor types. Five of the six lung cancer cases (83\%) smoked. Regarding possible carcinogenic agents, only one cancer patient received cyclophosphamide therapy and eight received methotrexate (MTX) or anti-TNF agents [22].

\section{Systemic Lupus Erythematosus}

In systemic lupus erythematosus (SLE), some small series and cohort studies suggested an increased risk for lymphomas, as well as for sarcomas and various types of cancer. In SLE, the general risk for cancer may not be increased, as the SIR of all malignancies is around 1.1-1.5. However, the risk of certain tumor types may be elevated in SLE. In recent cohorts, the SIR of non-Hodgkin's lymphomas was between 2.8 and 7.4. There may also be increased risk for lung, skin and hepatobiliary cancer in lupus. In contrast, the risk of cancer of the reproductive system is low $($ SIR $<1)$. The risk of malignancies in SLE may be independent of SLE clinical activity or organ involvement. Common genetic background and Epstein-Barr virus infection may be possible pathogenic factors in this respect. Older age and longer disease duration may be associated with elevated cancer risk in lupus [17, 18, 23-25]. 
We have recently analyzed data from 860 SLE patients in a study period between 1970 and 2004. Results were compared with the HFA database. A total of 37 patients (4.3\%) presented with malignancy. Cancer prevalence was the highest in the first 5-10 years of lupus. The most common types of malignancy were breast cancer $(n=11$; SIR 0.6), colorectal cancer ( $\mathrm{n}=5$; SIR 0.5), cervix cancer $(\mathrm{n}=5$; SIR 1.7), lymphoproliferative diseases $(\mathrm{n}=5$; SIR 1.3) and lung cancer $(\mathrm{n}=4$; SIR 0.5). The risk was the highest for non-Hodgkin's lymphoma (SIR 3.5). No correlation between cancer risk and immunosuppressive therapy could be identified. Cancer-related mortality was $11 \%[23]$.

\section{Systemic Sclerosis}

Patients with scleroderma are at an increased risk for developing malignancy in organs and tissues affected by fibrosis, as well as for B cell malignancies. Thus, lung, esophageal, skin and breast cancer and non-Hodgkin's lymphoma have been associated with systemic sclerosis (SSc). In various cohorts, scleroderma patients had a 1.5to 10.7-fold increased risk for these malignancies. Older age at the time of diagnosis and longer disease duration seem to be major risk factors for the development of cancer in SSc [17, 26-28].

Recently we have reported three cases of B cell lymphoma in our cohort of 218 scleroderma patients under follow-up. Chronic B cell stimulation is a crucial pathogenic factor in lymphoma development. Indeed, two cases of Zap $70^{+}$B-CLL and a chronic small lymphocytic B cell non-Hodgkin's lymphoma were diagnosed in patients with diffuse cutaneous SSc. Our data indicate that the incidence of lymphoma among Hungarian SSc patients may be 1.9-2.5 times higher than that in the general population. In our three patients, B cell lymphoma developed early, within 2 years after the onset of SSc [28].

We have also assessed cancer prevalence in $218 \mathrm{SSc}$ patients. We identified 10 cases of malignancy (4.6\%). Half of the SSc patients with cancer belonged to the diffuse cutaneous subtype. The mean age at the diagnosis of malignancy was 61.5 years. The mean disease duration of scleroderma at the time of cancer diagnosis was 6.6 years. Five patients died, four due to the underlying malignancy. Among the five surviving patients, the mean survival time was 4.9 years. Regarding types of malignancies, three patients had non-Hodgkin's lymphoma (SIR 43.4), two had lung cancer (SIR 8.6), two had breast cancer (SIR 5.8), one had leiomyosarcoma of the leg (SIR 19.8), one had esophageal cancer (SIR 52.4), one had cervix cancer (SIR 37.0) and one had skin cancer (SIR 26.6). In com- parison to the HFA database, the overall SIR of all malignancies in SSc was 1.07, varying between 5.8 and 52.4 in different tumor types. Only one cancer patient received cyclophosphamide therapy [29].

\section{Immunosuppressive, Biological Agents and Tumor Development}

\section{General Considerations}

Numerous immunosuppressive drugs and, recently, biological agents have been used to treat arthritis and autoimmune diseases. Some of these agents may directly or indirectly be associated with the subsequent development of malignancies. Such drugs may confer the risk through direct DNA mutagenesis, generalized immunosuppression increasing the risk of virus-associated lymphomas or through toxic injury as seen in the urinary bladder when using cyclophosphamide. Longer treatment has been associated with increasing the risk of tumorigenesis $[1,30$ 32]. Among traditional immunosuppressive drugs and biologics, only cyclophosphamide was found to be definitely carcinogenic. There has been significant controversy regarding MTX, azathioprine or anti-TNF biologics in this respect [30-33]. It is very likely, that 'old drugs' used to treat RA in the 1970s and 1980s, such as cyclophosphamide or chlorambucil, may slightly increase the risk of malignancies (with a relative risk of 1.5), while newer agents, such as MTX, leflunomide or biologics may not $[31,33]$.

\section{Traditional Immunosuppressive Drugs}

The use of cyclophosphamide in RA may result in a 1.5- to 4-fold risk of developing bladder or skin cancer, as well as lymphoproliferative diseases. Risk factors include higher total dose, long-term treatment and tobacco use. Recently, cyclophosphamide has only been rarely used in RA. The carcinogenic effect of cyclophosphamide could not be confirmed in SLE and SSc [31,33].

MTX, the gold standard in the treatment of RA, generally has a favorable side-effect profile. There has been no strong evidence suggesting that MTX would increase the risk for the development of solid tumors. In contrast, some earlier reports suggested that MTX might be related to the development of lymphomas; however, this has not been confirmed in recent large studies. As RA itself may slightly increase the risk for lymphomas, it is difficult to assess whether MTX treatment would favorably or unfa- 
vorably affect the association of lymphoproliferative diseases with RA. Yet, sustained clinical activity of RA may be the primary risk factor for secondary malignancies and MTX may have a net beneficial effect in this respect [32-34].

Azathioprine has been associated with an increased risk of both lymphoma and solid tumors in early RA cohorts; however, recent studies have not confirmed this. Azathioprine does not seem to increase lymphoma risk in recent SLE cohorts [32-34].

Although cyclosporine A has been used to treat RA since the early 1980s, there have not been enough patients followed longitudinally to determine whether cyclosporine A would convey an increased risk for malignancies. In retrospective analyses of RA patients treated with cyclosporine $\mathrm{A}$, increased risk for malignancies was not observed $[33,34]$.

In the case of leflunomide, it does not appear that this drug would increase the risk of any malignancies $[33,34]$.

\section{Biologic Agents}

There has been a debate whether biological agents, predominantly TNF inhibitors, would or would not increase the risk for the development of malignancies, primarily lymphomas. In the early, pre-biologic era, a close correlation between sustained RA clinical activity and lymphoma risk was determined. In early studies using biological agents, anti-TNF therapy resulted in a slight increase of hematological malignancies (OR 2.1) in comparison to hospital registry data (OR 1.7). However, biological therapy is introduced to RA patients with more active disease [20,30,31]. Therefore, early studies could not determine whether biologics would have a direct effect on the development of malignancies or not.

Very recently, Askling et al. [30] assessed data of almost 68,000 RA patients in the ARTIS registry. Altogether 6,604 patients received biologics between 1999 and 2006. There were 26 cases of lymphoma among patients treated with biologics. The lymphoma risk was higher in biologic-treated patients in comparison to RA patients treated with conventional DMARDs (SIR 1.35) or versus the general population (SIR 2.7). However, RA patients starting anti-TNF therapy in 1998-2001 accounted for the entire increase in lymphoma risk versus the two comparators. By contrast, lymphoma risk did not vary significantly by time since the start of first treatment, with the accumulated duration of treatment or with the type of anti-TNF agent. Thus, the increased lymphoma risk is mostly attributed to early years. In recent years, TNF antagonists were not associated with any major further increase in the already elevated lymphoma occurrence in RA. Thus, as many recent cohorts confirmed, it is very likely that RA itself, not anti-TNF therapy, is responsible for increased lymphoma risk in RA [20,30].

Regarding other biologics, there is no new signal suggesting that abatacept would increase lymphoma or cancer risk $[20,30]$.

Some biologics may even have a favorable influence on the risk of malignancies. For example, rituximab attenuated the development of MALT lymphoma in SS [19]. There have been no published data on the relationship of new biologics, such as tocilizumab, golimumab or certolizumab pegol to cancer risk.

\section{Soluble Tumor Antigens in Rheumatic Diseases}

There have been scattered reports, that some tumorassociated antigens (TAA) may, apart from cancer cells, become expressed on the surface of inflammatory cells. We and others have detected carcinoembryonic antigen (CEA)-related antigens (CD66b and CD66c) on neutrophils and monocyte/macrophages. In addition, there is an increased expression of CD66 in the RA synovial tissue in comparison to normal synovia $[1,35]$. Among tumor antigens, CEA is present mostly on colorectal and gastric carcinomas, CA 15-3 on breast carcinoma, CA 19-9 on pancreatic carcinoma, CA 125 on ovarian carcinoma and CA 72-4 on gastric and mucinous ovarian carcinoma. Members of the CD66 family of TAAs, as well as CA 19-9, CA 125 (MUC16) and CA 15-3 (MUC1) may serve as cell adhesion molecules as they contain carbohydrate determinants. Thus, these TAAs are involved in the adhesive properties of tumor cells and possibly inflammatory leukocytes $[1,36]$.

\section{Soluble TAAs in RA, SLE and SSc}

We and others have detected increased production of CEA and CA 19-9 in RA $[1,35,37]$. Also, increased production of CA 125, CA 15-3 and CA 19-9 was detected in SSc and SLE patients $[37,38]$. We have recently assessed levels of TAAs including CEA, CA 125, CA 15-3, CA 19-9 and CA 72-4 in the sera of RA, SLE and SSc patients, as well as healthy subjects. There were significantly more RA patients showing abnormally high levels of CA 125, CA 19-9 and CA 15-3 in comparison to controls. The mean absolute serum levels of CA 125 and CA 19-9 were also significantly higher in RA compared to controls. In 
RA, serum CEA showed a significant correlation with IgM rheumatoid factor. There were significantly more SSc patients showing abnormally high levels of CA 19-9, CA 125 and CA 15-3 in comparison to controls. In SLE, significantly more patients had elevated levels of CEA, CA 19-9, CA 125 and CA 72-4 than did controls. The mean absolute serum levels of CEA and CA 15-3 were also significantly higher in SSc compared to controls. Among SSc patients, serum CEA, CA 15-3 and CA 19-9 significantly correlated with renal involvement. Serum CA 15-3 also correlated with joint involvement, antinuclear antibody positivity and CRP levels. In SLE, serum CA 72-4 correlated with central nervous involvement and CA 125 correlated with the SLEDAI composite activity index. Thus, there may be increased production of various TAAs in RA, SSc and SLE. These TAAs contain carbohydrate motifs and, thus, they may be involved in synovitis-associated adhesive events. Furthermore, some TAAs may also correlate with disease activity, organ involvement and prognostic factors in these diseases $[1,39$, 40].

\section{Conclusions}

With the development of modern therapy and better care of patients with autoimmune rheumatic diseases, there has been an increased survival of patients. Thus, chronic comorbidities, such as malignancies have become a major issue in this context. It has been recognized that malignancies are associated with a wide range of rheumatic disorders and symptoms. Paraneoplastic symptoms may precede the diagnosis of the tumor, and can be predictive of a malignant disease. Numerous musculoskeletal diseases including connective tissue diseases, arthritides, metabolic and soft tissue disorders may be paraneoplasias. Various rheumatic diseases have been associated with the development of secondary tumors. Several pathogenic factors including common genes, viruses, sustained B cell activation and others have been implicated in carcinogenesis related to autoimmune diseases. Sometimes it is difficult to differentiate between paraneoplasias and malignancies secondary to autoimmune diseases. There have been controversies regarding cancer risk in various rheumatic diseases. While there is enough evidence for increased general cancer risk, as well as lymphoma and lung cancer risk in RA and scleroderma, the general risk of malignancies in SLE may not be increased. Early reports from the 1980s and 1990s suggested that traditional immunosuppressive agents and biologics might increase the risk of lymphoproliferative diseases. Yet, sustained systemic inflammation and disease activity may be primary risk factors for the development of malignancies. Recent studies from large cohorts did not confirm that MTX, azathioprine, leflunomide, cyclosporine A, anti-TNF agents or abatacept would increase the risk of lymphomas or solid tumors. In contrast, long-term cyclophosphamide therapy may still have carcinogenic effects. Finally, TAAs may serve as adhesion receptors expressed on inflammatory leukocytes, as well as on tumor cells. There is increased production of soluble TAAs of rheumatic diseases, which may be related to disease activity or organ involvement. All these data point out the importance of aggressive therapy and close follow-up of rheumatic patients at high risk for developing malignancies.

\section{References}

-1 Szekanecz E, Andras C, Sandor Z, AntalSzalmas P, Szanto J, Tamasi L, Kiss E, Szekanecz Z: Malignancies and soluble tumor antigens in rheumatic diseases. Autoimmun Rev 2006;6:42-47.

$>2$ Chakravarty E, Genovese MC: Rheumatic syndromes associated with malignancy. Curr Opin Rheumatol 2003;15:35-43.

$\checkmark 3$ Abu-Shakra M, Buskila D, Ehrenfeld M, Conrad K, Shoenfeld Y: Cancer and autoimmunity: autoimmune and rheumatic features in patients with malignancies. Ann Rheum Dis 2001;60:433-441.

4 Andras C, Csiki Z, Ponyi A, Illes A, Danko K: Paraneoplastic rheumatic syndromes. Rheumatol Int 2006;26:376-382. $\checkmark 5$ Bernatsky S, Ramsey-Goldman R, Clarke A: Malignancy and autoimmunity. Curr Opin Rheumatol 2006;18:129-134.

$\checkmark 6$ Andras C, Ponyi A, Constantin T, Csiki Z, Szekanecz E, Szodoray P, Danko K: Dermatomyositis and polymyositis associated with malignancy: a 21-year retrospective study. J Rheumatol 2008;35:438-444.

7 Launay D, Le Berre R, Hatron PY, Peyrat JP, Hachulla E, Devulder B, Hebbar M: Association between systemic sclerosis and breast cancer: eight new cases and review of the literature. Clin Rheumatol 2004;23:516-522.

8 Taillan B, Castanet J, Garnier G, Pesce A, Sanderson F, Fuzibet JG, Dujardin P: Paraneoplastic raynaud's phenomenon. Clin Rheumatol 1993;12:281-282.
9 Anton E: More on polymyalgia rheumatica (PMR) as a paraneoplastic rheumatic syndrome in the elderly (bicytopenia and PMR preceding acute myeloid leukemia). J Clin Rheumatol 2007;13:114.

$>10$ Perez NB, Bernad B, Narvaez J, Valverde J: Erythema nodosum and lung cancer. Joint Bone Spine 2006;73:336-337.

11 Stummvoll GH, Aringer M, Machold KP, Smolen JS, Raderer M: Cancer polyarthritis resembling rheumatoid arthritis as a first sign of hidden neoplasms. Report of two cases and review of the literature. Scand J Rheumatol 2001;30:40-44. 
12 Shih WJ: Pulmonary hypertrophic osteoarthropathy and its resolution. Semin Nucl Med 2004;34:159-163.

13 Cohen PR: Paraneoplastic relapsing polychondritis. Arch Dermatol 2007;143:949950.

14 Paira S, GrafC, Roverano S, Rossini J: Remitting seronegative symmetrical synovitis with pitting oedema: a study of 12 cases. Clin Rheumatol 2002;21:146-149.

15 Dahl PR, Su WP, Cullimore KC, Dicken CH: Pancreatic panniculitis. J Am Acad Dermatol 1995;33:413-417.

16 Kurzrock R, Cohen PR: Paraneoplastic erythromelalgia. Clin Dermatol 1993;11:7382.

17 Zintzaras E, Voulgarelis M, Moutsopoulos HM: The risk of lymphoma development in autoimmune diseases: a meta-analysis. Arch Intern Med 2005;165:2337-2344.

18 Toussirot E, Roudier J: Epstein-Barr virus in autoimmune diseases. Best Pract Res Clin Rheumatol 2008;22:883-896.

19 Pijpe J, van Imhoff GW, Spijkervet FK, Roodenburg JL, Wolbink GJ, Mansour K, Vissink A, Kallenberg CG, Bootsma H: Rituximab treatment in patients with primary Sjogren's syndrome: an open-label phase II study. Arthritis Rheum 2005;52:27402750.

20 Askling J: Malignancy and rheumatoid arthritis. Curr Rheumatol Rep 2007;9:421 426.

-21 Klareskog L, Padyukov L, Alfredsson L: Smoking as a trigger for inflammatory rheumatic diseases. Curr Opin Rheumatol 2007; 19:49-54.

22 Szekanecz É, Szűcs G, Kiss E, Szabó Z, Szántó S, Tarr T, Szekanecz Z: Secondary malignancies in rheumatoid arthritis (in Hungarian). Lege Artis Med 2008;18:886-892.
23 Tarr T, Gyorfy B, Szekanecz E, Bhattoa HP, Zeher M, Szegedi G, Kiss E: Occurrence of malignancies in Hungarian patients with systemic lupus erythematosus: results from a single center. Ann NY Acad Sci 2007;1108: 76-82.

24 Abu-Shakra M, Gladman DD, Urowitz MB: Malignancy in systemic lupus erythematosus. Arthritis Rheum 1996;39:1050-1054.

25 Bernatsky S, Ramsey-Goldman R, Clarke AE: Malignancy in systemic lupus erythematosus: what have we learned? Best Pract Res Clin Rheumatol 2009;23:539-547.

26 Abu-Shakra M, Guillemin F, Lee P: Cancer in systemic sclerosis. Arthritis Rheum 1993; 36:460-464.

27 Czirjak L, Kumanovics G, Varju C, Nagy Z, Pakozdi A, Szekanecz Z, Szucs G: Survival and causes of death in 366 Hungarian patients with systemic sclerosis. Ann Rheum Dis 2008;67:59-63.

28 Szekanecz E, Szamosi S, Gergely L, Keszthelyi P, Szekanecz Z, Szucs G: Incidence of lymphoma in systemic sclerosis: a retrospective analysis of 218 Hungarian patients with systemic sclerosis. Clin Rheumatol 2008;27: 1163-1166.

29 Szekanecz É, Szekanecz Z, Kiss E, Keszthelyi P, Szücs G: Secondary malignancies in systemic sclerosis (in Hungarian). Hung Rheumatol 2009;50:4-9.

30 Askling J, Bongartz T: Malignancy and biologic therapy in rheumatoid arthritis. Curr Opin Rheumatol 2008;20:334-339.

31 Asten P, Barrett J, Symmons D: Risk of developing certain malignancies is related to duration of immunosuppressive drug exposure in patients with rheumatic diseases. J Rheumatol 1999;26:1705-1714.

32 Beauparlant P, Papp K, Haraoui B: The incidence of cancer associated with the treatment of rheumatoid arthritis. Semin Arthritis Rheum 1999;29:148-158.

33 Jones M, Symmons D, Finn J, Wolfe F: Does exposure to immunosuppressive therapy increase the 10 year malignancy and mortality risks in rheumatoid arthritis? A matched cohort study. Br J Rheumatol 1996;35:738-745.
34 Bernatsky S, Clarke AE, Suissa S: Hematologic malignant neoplasms after drug exposure in rheumatoid arthritis. Arch Intern Med 2008;168:378-381.

35 Szekanecz Z, Haines GK, Harlow LA, Shah MR, Fong TW, Fu R, Lin SJ, Koch AE: Increased synovial expression of the adhesion molecules cd66a, cd66b, and cd31 in rheumatoid and osteoarthritis. Clin Immunol Immunopathol 1995;76:180-186.

36 Lance MP: CEA as a cell adhesion molecule. Gastroenterology 1990;99:277-278.

- 37 Shimomura C, Eguchi K, Kawakami A, Migita K, Nakao H, Otsubo T, Ueki Y, Tezuka $\mathrm{H}$, Yamashita S, Matsunaga M, et al.: Elevation of a tumor associated antigen CA 19-9 levels in patients with rheumatic diseases. J Rheumatol 1989;16:1410-1415.

- 38 Takeda N, Ihn H, Teramoto S: Markedly increased levels of IL- 6 and CA125 in pleural fluid of an elderly person with overlap syndrome of systemic sclerosis and systemic lupus erythematosus. Age Ageing 2001;30:171.

39 Szekanecz E, Szucs G, Szekanecz Z, Tarr T, Antal-Szalmas P, Szamosi S, Szanto J, Kiss E: Tumor-associated antigens in systemic sclerosis and systemic lupus erythematosus: associations with organ manifestations, immunolaboratory markers and disease activity indices. J Autoimmun 2008;31:372376.

40 Szekanecz E, Sandor Z, Antal-Szalmas P, Soos L, Lakos G, Besenyei T, Szentpetery A, Simkovics E, Szanto J, Kiss E, Koch AE, Szekanecz Z: Increased production of the soluble tumor-associated antigens CA 19-9, CA 125 , and CA $15-3$ in rheumatoid arthritis: potential adhesion molecules in synovial inflammation? Ann NY Acad Sci 2007;1108: 359-371. 